\title{
Efeito da estrutura de propriedade na relação entre assimetria de informação e política de pagamentos
}

\author{
Ownership structure effect on relationship between information asymmetry and payment policy \\ Efecto de la estructura de propiedad sobre la relación entre asimetría de información y política de
}

pagos

Recebido: 21/04/2021 | Revisado: 29/04/2021 | Aceito: 01/05/2021 | Publicado: 15/05/2021

\author{
Erica Juvercina Sobrinho \\ ORCID: https://orcid.org/0000-0001-9196-3184 \\ Universidade Federal de Uberlândia, Brasil \\ E-mail: ericajuvercina@hotmail.com \\ Vitor Borges Tavares \\ ORCID: https://orcid.org/0000-0002-7234-3761 \\ Universidade Federal de Uberlândia, Brasil \\ E-mail: vitorbtavares@gmail.com \\ Pablo Rogers Silva \\ ORCID: https://orcid.org/0000-0002-0093-3834 \\ Universidade Federal de Uberlândia, Brasil \\ E-mail: pablorogers@ufu.br
}

\begin{abstract}
Resumo
A definição de uma política de pagamentos aos acionistas depende de um arcabouço de decisões no âmbito corporativo, o que pode gerar conflitos de interesses. Nesse contexto, o presente estudo analisou o efeito moderador da estrutura de propriedade na relação entre assimetria de informação e a política de pagamento de dividendos das empresas brasileiras de capital aberto. Os resultados não rejeitam a hipótese (H1) de que a assimetria de informação afeta positivamente a remuneração ao acionista. Em relação ao efeito moderador da estrutura de propriedade (H2), os resultados não foram consensuais, pois a estrutura de propriedade moderou a relação entre assimetria e política de pagamentos em apenas uma das duas proxies de assimetria de informação. Dessa forma, esta pesquisa contribui com a literatura e com os interessados no mercado de ações brasileiro ao apresentar, de diferentes perspectivas, o efeito moderador da estrutura de propriedade na relação entre assimetria de informação e remuneração ao acionista.
\end{abstract}

Palavras-chave: Política de pagamentos; Assimetria de informação; Estrutura acionária.

\begin{abstract}
Defining a shareholder payment policy depends on a corporate decision-making framework, or what may lead to conflicts of interest. In this context, the present study analyzes the moderating effect of ownership structure on the relationship between information asymmetry and payment policy of publicly traded Brazilian companies. The results do not reject the hypothesis (H1) that information asymmetry positively affects compensation when triggering. Regarding the moderating effect of ownership structure (H2), the results were not consensual, as ownership structure moderated the relationship between asymmetry and payment policy in only one of the two information asymmetry proxies. Thus, this research contributes to literature and stakeholders in the Brazilian stock market, presenting different perspectives, or moderating effect of ownership structure on the relationship between information asymmetry and shareholder valuation.
\end{abstract}

Keywords: Payment policy; Information asymmetry; Shareholding structure.

\section{Resumen}

La definición de una política de pagos a accionistas depende de un marco de decisión a nivel corporativo, que puede generar conflictos de interés. En este contexto, el presente estudio analizó el efecto moderador de la estructura de propiedad sobre la relación entre la asimetría de información y la política de pago de dividendos de las empresas brasileñas que cotizan en bolsa. Los resultados no rechazan la hipótesis (H1) de que la asimetría de información afecta positivamente la retribución al accionista. En cuanto al efecto moderador de la estructura de propiedad (H2), los resultados no fueron consensuados, ya que la estructura de propiedad moderó la relación entre asimetría y política de pago en solo uno de los dos proxies de asimetría de información. Así, esta investigación contribuye a la literatura y a los interesados en el mercado de valores brasileño al presentar, desde diferentes perspectivas, el efecto moderador de la estructura de propiedad sobre la relación entre asimetría de información y remuneración al accionista.

Palabras clave: Política de pagos; Asimetría de información; Estructura accionarial. 


\section{Introdução}

A definição de uma política de pagamentos aos acionistas depende de um arcabouço de decisões no âmbito corporativo, o que pode gerar conflitos de interesses. Por isso, é importante entender os fatores que influenciam as decisões de distribuição e retenção de caixa para gestores financeiros, investidores e para os órgãos reguladores de mercado (Forti, Peixoto \& Alves, 2015).

Nesse contexto, desde a teoria da irrelevância dos dividendos (Miller \& Modigliani, 1961; Black \& Scholes, 1974; Benartzi, Michaely \& Thaler, 1997), até sua contraposição pela teoria da relevância dos dividendos (Gordon, 1959; Lintner, 1962), a busca pelas implicações de uma política de pagamentos aos acionistas centraliza-se em seus efeitos informacionais ao mercado, como pela teoria da sinalização (Spence, 1973; Bhattacharya, 1979), como pela teoria da agência (Jensen \& Meckling, 1976; Rozeff, 1982). Marques et al. (2020) mapeiam essas teorias aplicadas no mercado brasileiro de capitais nos últimos 11 anos e concluem que elas têm sido utilizadas de forma uniforme, sendo todas elas confirmadas no contexto brasileiro. Os autores encontraram 11 artigos elaborados e publicados versando cada uma dessas teorias e indicaram que existem poucas divergências a respeito do tema entre as pesquisas, sendo a maioria confirmadas no contexto nacional.

No Brasil, diferentemente de quase todos os outros países (Futema, Basso \& Kayo, 2009), a legislação (Lei no 6.404/1976) prevê que, via de regra, no mínimo $25 \%$ dos lucros ajustados sejam destinados à distribuição de dividendos. Como alternativa, o que representa outra característica peculiar do mercado brasileiro, a Lei $n^{\circ}$ 9.249/1995 permitiu a possibilidade de remuneração ao acionista por meio de juros sobre o capital próprio (JSCP).

A remuneração aos acionistas via JSCP possibilita vantagens fiscais à empresa, sendo dedutível da base de cálculo para o imposto de renda pago pela empresa, mas tributada para o acionista, enquanto os dividendos são tributados no nível corporativo, não sendo tributados no nível do acionista (Boulton et al., 2012). Desse modo, a escolha entre distribuir a maior parte dos lucros no formato de dividendos e/ou juros sobre o capital próprio é influenciada por diversos fatores (Mota \& Junior, 2007), mas os gestores preferem pagar dividendos do que juros sobre capital próprio (Boulton, Braga-Alves, \& Shastri, 2012).

Dessa forma, alguns estudos analisaram fatores relevantes no processo decisório de pagamento de proventos aos acionistas (Heineberg \& Procianoy, 2003; Silva, 2004; Iquiapaza, Lamounier, \& Amaral, 2006; Mota \& Junior, 2007; Ferreira Jr, Nakamura, Martin, \& Bastos, 2010; Forti, Peixoto \& Alves, 2015; Forti \& Schiozer, 2015; Rosa, Araújo \& Rogers, 2021). Porém, tais pesquisas não aprofundaram na questão da estrutura de propriedade e assimetria informacional.

Essas considerações são relevantes, pois as regras brasileiras para dividendos obrigatórios ajudam a proteger os direitos de fluxo de caixa dos acionistas minoritários, sem distorcer significativamente os planos de investimento corporativos (Martins \& Novaes, 2012). Paralelamente, gera obstáculos aos acionistas controladores com quantidade reduzida de recursos em caixa (La Porta, Lopez-De-Silanes, Shleifer, \& Vishny, 2000; Vancin \& Procianoy, 2016). Assim, a concentração de propriedade acionária possui relevante importância na política de distribuição de lucros (Holanda \& Coelho, 2012).

Ao mesmo tempo, em um mercado emergente, a assimetria de informação desempenha papel de liderança na explicação do comportamento dos pagamentos de dividendos (Ofer \& Siegel, 1987; Rajverma, Misra, Mohapatra, \& Chandra, 2019). Nesse sentido, os dividendos também podem ser usados para fins de minimização de problemas de agência (Lin et al., 2017). Outrossim, de acordo com a hipótese de sinalização dos dividendos (Spence, 1973), os tomadores de decisão no âmbito corporativo possuem informações privilegiadas (Procianoy \& Verdi, 2009), sugerindo que acionistas controladores possuem benefícios e expropriam os acionistas minoritários (Lee \& Xiao, 2011). Dessa maneira, a remuneração ao acionista pode ser utilizada para estabelecer tratamento adequado aos acionistas minoritários (Silva, 2004).

Assim sendo, este trabalho analisa o efeito moderador da estrutura de propriedade na relação entre assimetria de informação e a política de pagamento aos acionistas das empresas brasileiros de capital aberto. Desse modo, o estudo contribui 
com a literatura e com os interessados no mercado de ações brasileiro ao apresentar, de diferentes perspectivas, a associação entre estrutura de propriedade, assimetria de informação e política de pagamentos.

Este estudo se diferencia das demais pesquisas sobre estrutura de propriedade no Brasil por duas razões. Primeiro, refere-se ao emprego de regressão linear múltipla em um painel de 22 anos. Segundo, corresponde à utilização de proxies para captar o efeito moderador na política de pagamentos envolvendo o pagamento tanto de dividendos como também de juros sobre capital próprio. As demais pesquisas não utilizaram uma janela temporal tão longa nem tentaram demonstrar o efeito moderador nos dois formatos possíveis na política brasileira de pagamentos ao investidor. Por fim, pode apontar uma consistência no padrão de pagamentos de dividendos com o intuito de minimizar as reações adversas dos acionistas.

No que tange ao embasamento teórico, esta pesquisa parti do princípio de que definir a política de dividendos é uma das principais tomadas de decisão da empresa (Lin, Chen, \& Tsai, 2017). Ela permite às companhias transferir parte do fluxo de caixa gerado por seus negócios aos investidores e acionistas (Kumar \& Vergara-Alert, 2018). No entanto, a remuneração aos acionistas, de parte dos lucros obtidos, pode prejudicar o fluxo de caixa e as possibilidades de investimento da empresa (Topak \& Dereli, 2018).

Nesse contexto, a política de dividendos sinaliza informações sobre o desempenho da empresa para os participantes do mercado (Ofer \& Siegel, 1987). De fato, pela teoria da sinalização, os investidores correlacionam qualquer alteração no padrão de pagamento com a saúde e a gestão da empresa (Rajverma et al., 2019). Assim, os dividendos podem ser usados para transmitir informações positivas ao mercado, evidenciando que a empresa tem boa capacidade de geração de caixa. Entretanto, o pagamento de dividendos também podem ser visto de forma negativa pelo mercado, representando que a empresa não tem opções de investimento (Allen \& Michaely, 2003).

Para os acionistas, os dividendos não são apenas uma alternativa de renda, mas também são informações que influenciam a tomada de decisões de investimentos. A política de dividendos é um sinal que pode mudar as percepções dos investidores em relação às perspectivas de ganhos futuros da empresa (Ben-Nasr, 2015). Dessa forma, os gestores, muitas vezes relutantes em cortar dividendos para evitar declínio nos preços das ações (Rajverma et al., 2019), tentam estabelecer uma política ótima de dividendos que maximize o valor da empresa para não gerar efeito negativo nas metas futuras de investimento e no fluxo de caixa da empresa (Topak \& Dereli, 2018).

Não obstante, os dividendos também podem ser usados como ferramenta para minimizar problemas de agência (Lin et al., 2017), haja vista o conflito de interesses existente entre acionistas e gestores (Jensen \& Meckling, 1976). Por exemplo, maior pagamento de dividendos pode ser considerado um meio de estabelecer um tratamento adequado aos acionistas minoritários (Silva, 2004). Nesse sentido, a política de dividendos atenua a assimetria de informação entre acionistas e gestores (Moreiras, Tambosi Filho, \& Garcia, 2012).

Considerando que os gerentes têm mais informações do que os acionistas a respeito da situação da empresa (Moreiras et al., 2012), o pagamento de dividendos poderia ser utilizado para reduzir conflitos de agência, diminuindo a quantidade de recursos disponíveis aos gestores (Martins \& Famá, 2012).

Outrossim, o mercado de ações brasileiro contempla outro problema de agência, ainda mais acentuado, qual seja, o conflito de interesses entre acionistas maioritários e minoritários (Martins \& Famá, 2012). Nesse cenário, os controladores podem expropriar os minoritários, restringindo a transparência corporativa, visando obter benefícios próprios (Leuz, Nanda \& Wysocki, 2003; Lin, Chen \& Tsai, 2017), uma vez que os acionistas majoritários podem exercer forte influência sobre as decisões dos gestores (La Porta et al., 2000).

Por isso, tendo em vista que características acionárias da companhia são relevantes para a política de pagamento de dividendos (Duqi, Jaafar, \& Warsame, 2019), estabelecer a obrigatoriedade de distribuição de dividendos representa uma forma de proteger os direitos dos acionistas minoritários referentes ao fluxo de caixa das empresas (Martins \& Novaes, 2012). 
A legislação brasileira determina a obrigatoriedade de pagamento de dividendos para companhias abertas, rara no contexto mundial dos mercados de ações (Futema, Basso, \& Kayo, 2009). Contudo, algumas empresas usam brechas da lei para evitar o pagamento. Mas, apesar das brechas, a obrigatoriedade estabelecida em lei é eficaz no sentido de garantir maiores taxas de pagamento de dividendos (Martins \& Novaes, 2012).

Além disso, o Brasil tem outra característica distinta da maioria dos outros mercados de capitais. No país, o pagamento de recursos financeiros ao investidor pode ser feito na forma de juros sobre capital próprio (JSCP) ou de dividendos (Boulton et al., 2012). A remuneração do acionista via JSCP é considerada despesa financeira, sendo, diferentemente dos dividendos, dedutível para fins do cálculo do imposto de renda e contribuição social incidentes sobre os lucros pela empresa (Santos, 2007).

Desse modo, no âmbito empresarial, o pagamento de JSCP apresenta vantagens sobre o pagamento de dividendos, para fins de obtenção de melhor resultado financeiro, haja vista que o primeiro permite a dedução nos impostos (Boulton et al., 2012). Com efeito, aproveitar os benefícios relacionados ao JSCP é um desafio aos gestores (Rajverma et al., 2019).

Ademais, a escolha entre distribuir a maior parte dos lucros no formato de dividendos e/ou juros sobre o capital próprio é influenciada por diversos fatores. No Brasil, houve aumento na remuneração aos acionistas de 1999 a 2005, sendo o pagamento via dividendos a forma predominante (Mota, 2007). Desse modo, embora o pagamento de JSCP apresente vantagens fiscais sobre os dividendos, as empresas brasileiras continuam pagando dividendos para atender às demandas dos acionistas (Boulton et al., 2012).

Dessa forma, a possibilidade de pagamento via JSCP pode, ainda, acentuar o conflito entre os acionistas majoritários e minoritários, pois os controladores podem priorizar o pagamento via JSCP visando menor saída de recursos da companhia. Assim sendo, as características do mercado de ações brasileiro indicam que a concentração acionária tem efeito mediador na relação entre assimetria de informação e política de pagamentos.

Ante o contexto do mercado brasileiro, a primeira hipótese do estudo estabelecida $\left(H_{l}\right)$ considera que as empresas com maior assimetria de informação utilizam a política de pagamento como forma de diminuir os recursos à disposição dos gestores e atenuar esse conflito de agência (Moreiras et al., 2012). Logo:

H1 - Existe relação positiva entre assimetria de informação e a remuneração do acionista por meio de dividendos e JSCP.

No entanto, empresas com maior concentração acionária, expropriando os acionistas minoritários, que têm preferência pelo recebimento de dividendos (La Porta et al, 2000), remunerariam menos os acionistas para manter mais recursos sob seu controle (Silva, 2004; Vancin \& Procianoy, 2016). Os acionistas controladores, normalmente, têm poder sobre as empresas que excedem significativamente seus direitos de fluxo de caixa, principalmente por meio do uso de pirâmides e participação na administração (Porta, Lopez-de-Silanes, \& Shleifer, 1999).

A forte proteção ao acionista minoritário limita a capacidade dos tomadores de decisão dentro da empresa de adquirir benefícios de controle privado (Leuz et al., 2003). Em resposta, os acionistas controladores, em geral, não amparam as reformas em prol das minorias, mas sim realizam lobby em posição contrária (Porta et al., 1999; Silva, 2004). Como consequência, as empresas pagam dividendos baixos para abrir caminho aos acionistas controladores no desvio de ativos corporativos (La Porta et al., 2000).

A estrutura de propriedade acionária é importante na explicação da política de dividendos (Al-Najjar, 2011; Ben-Nasr, 2015; Duqi et al., 2019; Gugler, 2003; Ramli, 2010; Wei, Zhang, \& Xiao, 2004) e relevante para a assimetria entre grandes e pequenos acionistas (Huddart, 1993; Maug, 1998; Ramli, 2010). Com efeito, tem-se a outra hipótese da pesquisa $\left(H_{2}\right)$ : 
$\mathbf{H}_{2}$ - A estrutura de propriedade possui efeito moderador na relação entre assimetria de informação e política de pagamentos da empresa.

Esta pesquisa está estruturada em quatro seções. Na sequência desta introdução, foram evidenciados os métodos e dados utilizados para realizações dos testes propostos. Na sequência, os resultados foram apresentados e discutidos. Por fim, na quarta seção, foram tecidas as considerações finais.

\section{Metodologia}

Do ponto de vista metodológico, esta pesquisa é classificada como quantitativa, uma vez que, para produção de resultados, utiliza técnicas estatísticas aplicadas a um conjunto de dados (Pereira et al., 2018). A base de dados refere-se às empresas brasileiras de capital aberto na Bolsa B3, disponível na Economática ${ }^{\circledR}$, com 15848 observações ano-empresa dos relatórios financeiros no período entre 1996 a 2018, com exclusão do setor financeiro. O corte temporal se justifica por ser o período de vigência das duas alternativas de pagamento de proventos aos acionistas (dividendos e JSCP). Para corrigir possíveis problemas com outliers, houve winsorização dos dados a $1 \%$. Na Tabela 1 tem-se as variáveis consideradas no estudo.

As variáveis apresentadas compõem três modelos econométricos principais, equações (1), (2) e (3), com a utilização das quatro variáveis dependentes $\left(\mathrm{Y}_{\mathrm{it}}\right)$ já discriminadas $(D Y, J S C P, P A G T T$ e $D I F)$ e resultados provenientes de regressões em dados em painel.

Tabela 1. Variáveis da pesquisa.

\begin{tabular}{|c|c|c|c|c|}
\hline Variável & Fórmula & $\begin{array}{c}\text { Sinal } \\
\text { Esperado }\end{array}$ & Descrição & Literatura \\
\hline DY & $D Y_{\text {it }}=\frac{P D I V_{\text {it }}}{P A C A O_{\text {it }}}$ & & $\begin{array}{l}\text { Dividend Yield - pagamento de } \\
\text { dividendo por ação da empresa } \\
\text { dividido pelo preço da ação }\end{array}$ & Ferreira Jr et al., 2010 \\
\hline JSCP & $j S C P_{\text {it }}=\frac{P J S C P_{\text {it }}}{P A C A O_{\text {it }}}$ & & $\begin{array}{l}\text { Juros sobre capital próprio - } \\
\text { pagamento de juros sobre capital } \\
\text { próprio dividido pelo preço da } \\
\text { ação }\end{array}$ & Tomaz, 2017 \\
\hline PAGTT & $P A G T T_{\text {it }}=D Y_{i t}+J S C P_{\text {it }}$ & & $\begin{array}{l}\text { Pagamento total - dividend yield } \\
\text { mais juros sobre capital próprio }\end{array}$ & Mota \& Junior, 2007 \\
\hline DIF & $D v j S C P_{\text {it }}=D Y_{\text {it }}-j S C P_{\text {it }}$ & & $\begin{array}{l}\text { Diferença entre dividend yield e } \\
\text { juros sobre capital próprio, } \\
\text { possibilitando analisar como as } \\
\text { variáveis afetam a decisão entre o } \\
\text { pagamento de dividendos e o } \\
\text { pagamento de juros sobre o capital } \\
\text { próprio }\end{array}$ & *Proposição desta pesquisa \\
\hline EXP & $E X P_{\text {it }}=C O N_{\text {it }}-P R O P_{\text {it }}$ & + & $\begin{array}{l}\text { Grau de expropriação do } \\
\text { acionista - diferença entre o } \\
\text { percentual de ações ordinárias } \\
\text { (CON) e o percentual do total de } \\
\text { ações em posse do acionista } \\
\text { controlador (PROP) }\end{array}$ & $\begin{array}{l}\text { Dalmácio, Nossa, \& Filho, } \\
\text { 2007; Iquiapaza et al., 2006; } \\
\text { Loss \& Sarlo Neto, 2003; Mota } \\
\text { \& Junior, 2007; Procianoy \& } \\
\text { Vancin, 2014; Ramli, 2010; } \\
\text { Silveira, 2004; Wei et al., } 2004\end{array}$ \\
\hline ASY1 & $A S Y 1_{\text {it }}=100 * \frac{p}{P} * \sqrt{\frac{n}{N} * \frac{V}{V}}$ & + & $\begin{array}{l}\text { Liquidez da ação - volume de } \\
\text { negociação anual da ação em } \\
\text { bolsa de valores }\end{array}$ & $\begin{array}{l}\text { Albanez \& Valle, 2009; } \\
\text { Grullon, Michaely, \& } \\
\text { Swaminathan, 2002; Lin et al., } \\
2017\end{array}$ \\
\hline
\end{tabular}




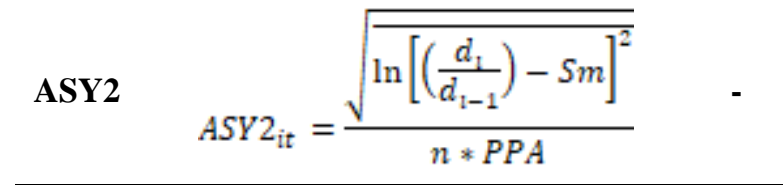

RISC $R I S C_{i t}=\frac{\operatorname{cov}\left(R i_{v} R m\right)}{\operatorname{var}(R m)}$

TAM $\quad T A M_{\text {it }}=\ln \left(A T_{\text {it }}\right)$

RENT $\quad R E N T_{\text {it }}=\frac{L O_{\text {it }}}{A T_{\text {it }}}$

OCRES OCRES it $=\frac{V M_{\text {it }}}{P L_{\text {it }}}$

LEMP $\quad L E M P_{\text {it }}=\frac{A C_{\text {it }}}{P C_{\text {it }}}$
Volatilidade da ação - desvio padrão do logaritmo neperiano das variações diárias da cotação de fechamento no período escolhido

Risco sistemático - medido pelo Beta da empresa, sendo covariância de retorno do ativo e retorno do mercado dividido pela variância do retorno do mercado

Tamanho da empresa logaritmo neperiano do ativo total da empresa

Rentabilidade - medida pelo retorno sobre o ativo (ROA), $+\quad$ sendo o lucro operacional (antes Desp. Financeiras) dividido pelo Ativo Total da empresa

Oportunidade de crescimento medida pelo market to book, sendo valor de mercado dividido pelo valor contábil da empresa

Liquidez geral da empresa ativo circulante dividido pelo passivo circulante
Albanez \& Valle, 2009; Halov \& Heider, 2011; Procianoy \& Vancin, 2014

Bernardo \& Ikeda, 2013; Forti et al., 2015; Lin et al., 2017; Ramli, 2010; Rozeff, 1982

Forti et al., 2015; Heineberg \& Procianoy, 2003; Iquiapaza et al., 2006; Lee \& Xiao, 2011; Mota \& Junior, 2007; Procianoy \& Vancin, 2014; Ramli, 2010; Wei et al., 2004

Forti et al., 2015; John, K., \& Knyazeva, 2006; Lee \& Xiao, 2011; Mota \& Junior, 2007

Forti et al., 2015; Heineberg \& Procianoy, 2003

Ferreira Jr et al., 2010; Forti et al., 2015; Silva, 2004; Viswanathan \& Acharya, 2011

DY, JSCP, PAGTT e DIF são as variáveis dependentes. EXP, ASY1 e ASY são as variáveis independentes de interesse e as outras são variáveis de controle. Fonte: Autores (2021).

$$
\begin{aligned}
& Y_{\text {it }}=\beta_{0}+\beta_{1} A S Y_{\text {it }}+\beta_{2} R I S C_{\text {it }}+\beta_{2} R E N T_{\text {it }}+\beta_{4} O C R E S_{\text {it }}+\beta_{5} T A M_{\text {it }}+\beta_{6} L E M P_{\text {it }}+w_{\text {it }} \\
& Y_{\text {it }}=\beta_{0}+\beta_{1} A S Y_{\text {it }}+\beta_{2} E X P_{\text {it }}+\beta_{3} \text { RISC }_{\text {it }}+\beta_{4} R E N T_{\text {it }}+\beta_{5} \text { OCRES } \text { it }+\beta_{6} \text { TAM }_{\text {it }}+\beta_{7} L E M P_{\text {it }}+w_{\text {it }} \\
& Y_{\text {it }}=\beta_{0}+\beta_{1} A S Y_{\text {it }}+\beta_{2} A S Y_{\text {it }} E X P_{\text {it }}+\beta_{3} R I S C_{\text {it }}+\beta_{4} R E N T_{\text {it }}+\beta_{5} O C R E S_{\text {it }}+\beta_{6} T_{A M}+\beta_{7} L E M P_{\text {it }}+w_{\text {it }}
\end{aligned}
$$

Para todas as equações, foram realizados os testes formais de definição do melhor modelo de dados em painel. Os testes de Chow e de Hausman evidenciaram, em todas equações, que o modelo de efeitos fixos era o mais adequado para a amostra. Assim, $w_{i t}=e_{i}+u_{i t}$ em que os componentes de erro individual ( $\left.e_{i}\right)$ não estão correlacionados entre si, nem com as unidades de corte transversal e de série temporal $\left(u_{i t}\right)$. Adicionalmente, $w_{\text {it }}$ não está correlacionado com qualquer uma das variáveis explanatórias incluídas no modelo. Como resultado dos pressupostos estabelecidos segue que $E\left(w_{\text {it }}\right)=0$ e $\operatorname{var}\left(w_{i t}\right)=\sigma_{e}^{2}+\sigma_{u}^{2}$, que no caso do modelo de efeitos fixos tem-se $\sigma_{e}^{2}=0$.

Ademais, em todas as equações foram testados a multicolinearidade (via fator de inflação da variância - VIF), a heterocedasticidade (Teste de Wald) e a autocorrelação (Teste de Wooldridge). A heteroscedasticidade, verificada em todos os modelos, foi tratada por meio de estimação dos parâmetros com erros robustos. Da mesma forma, a autocorrelação, 
identificada em alguns modelos, foi corrigida. No caso de autocorrelação, realizou-se a estimação por meio da estatística de Driscoll-Kraay, eficaz para corrigir problemas de heterocedasticidade e autocorrelação em regressões com dados em painel utilizando efeitos fixos (Vogelsang, 2012).

\section{Resultados e discussão}

Na Tabela 2 constam as estatísticas descritivas para as variáveis utilizadas nos modelos. Nas Tabelas 3 e 4 são apresentados os resultados dos modelos econométricos em que a liquidez da ação foi proxy da assimetria de informação. Nas Tabelas 5 e 6, evidencia-se os resultados dos testes realizados com a assimetria sendo medida pela volatilidade das ações.

A Tabela 2 mostra que, na média, as empresas pagaram pouco mais que o dobro de dividendos em comparação com JSCP no período de análise. Esses dados corroboram com o fato de que embora o pagamento de JSCP apresente vantagens fiscais sobre os dividendos, as empresas brasileiras continuam pagando dividendos para atender às demandas dos acionistas (Boulton et al., 2012). No entanto, algumas empresas pagaram mais JSCP do que dividendos anualmente, sendo 7.427 a maior diferença entre o valor pago por meio de JSCP por ação e o valor de dividendos pagos por ação por uma empresa no ano.

Tabela 2. Estatística descritiva para as variáveis.

\begin{tabular}{llllll}
\hline \multicolumn{1}{c}{ Variável } & Observações & \multicolumn{1}{c}{ Média } & Desvio padrão & \multicolumn{1}{c}{ Mínimo } & \multicolumn{1}{c}{ Máximo } \\
\hline DY & 3,423 & 5.718 & 7.015 & 0.041 & 43.229 \\
JSCP & 1,269 & 2.615 & 2.405 & 0.147 & 14.648 \\
PAGTT & 3,779 & 6.029 & 6.610 & 0.083 & 39.997 \\
DIF & 3,778 & 4.302 & 7.175 & -7.427 & 38.773 \\
ASY1 & 6,958 & 0.152 & 0.399 & 0.000 & 2.551 \\
ASY2 & 4,757 & 45.385 & 26.764 & 11.413 & 157.342 \\
EXP & 6,708 & 20.634 & 22.821 & 0.000 & 93.826 \\
RISC & 4,757 & 0.621 & 0.772 & -1.396 & 3.220 \\
RENT & 8,699 & -25.013 & 146.381 & -1187.34 & 40.926 \\
OCRES & 5,535 & 1.987 & 3.045 & 0.070 & 22.033 \\
TAM & 8,816 & 5.991 & 1.193 & 1.292 & 8.049 \\
LEMP & 8,724 & 3.666 & 13.838 & 0.008 & 123.555 \\
\hline
\end{tabular}

Notas: DY = dividendo pago por ação no ano; JSCP = JSCP pago por ação no ano; PAGTT = pagamento total (dividendo + JSCP) por ação no ano; DIF = diferença entre o dividendo pago por ação no ano e o JSCP pago por ação no ano; ASY1 = liquidez da ação no ano (proxy); ASY2 = volatilidade da ação no ano (proxy); EXP = estrutura de propriedade acionária no fim do ano; RISC = risco (beta) da ação no ano; RENT = rentabilidade (ROA) da empresa no ano; OCRES = oportunidade de crescimento (market to book) da empresa no ano; TAM = tamanho da empresa no fim do ano; LEMP = liquidez da empresa no ano. Fonte: Autores (2021). 
Tabela 3. Resultados para pagamento de dividendos e JSCP - liquidez da ação como proxy de assimetria.

\begin{tabular}{|c|c|c|c|c|c|c|}
\hline \multirow[b]{2}{*}{ Variável } & \multicolumn{3}{|c|}{ Dividendos } & \multicolumn{3}{|c|}{ JSCP } \\
\hline & Modelo 1 & Modelo 2 & Modelo 3 & Modelo 1 & Modelo 2 & Modelo 3 \\
\hline ASY1 & $-0.891 *$ & $-0.884 *$ & -0.246 & $-0.887 * *$ & $-1.076^{* *}$ & -0.587 \\
\hline EXP & & -0.020 & & & -0.031 & \\
\hline EXP*ASY1 & & & $-0.063^{*}$ & & & $-0.054 * * *$ \\
\hline RISCO & $-0.659 * * *$ & $-0.513 * *$ & $-0.491 *$ & -0.034 & -0.089 & -0.076 \\
\hline RENT & -0.037 & 0.060 & 0.060 & -0.012 & -0.011 & -0.011 \\
\hline OCRES & $-0.560 * * *$ & -0.524 & $-0.525 * * *$ & $-0.313 * * *$ & $-0.334 * * *$ & $-0.336^{* * *}$ \\
\hline TAM & $-2.946 * * *$ & $-2.782 * * *$ & $-2.860 * * *$ & $-2.333^{* *}$ & $-2.541 * *$ & $-2.431 * *$ \\
\hline LEMP & $-0.128 * *$ & $-0.081 * *$ & $-0.082 * *$ & -0.001 & -0.001 & -0.001 \\
\hline CONS & $27.312 * * *$ & $25.490 * * *$ & $25.668 * * *$ & $19.928 * *$ & $21.910 * * *$ & $20.749 * * *$ \\
\hline VIF (médio) & 1.27 & 1.27 & 1.49 & 1.34 & 1.32 & 1.67 \\
\hline Teste F & $9.00 * * *$ & $5.73 * * *$ & $6.24 * * *$ & $8.47 * * *$ & $5.62 * * *$ & $6.44 * * *$ \\
\hline $\mathrm{R}^{2}$ & 0.048 & 0.037 & 0.038 & 0.137 & 0.157 & 0.163 \\
\hline $\mathrm{n}$ & 2634 & 2190 & 2190 & 1163 & 1071 & 1071 \\
\hline Teste Chow & $3.16^{* * * *}$ & $3.36 * * *$ & $3.49 * * *$ & $3.31 * * *$ & $3.00 * * *$ & $3.12 * * *$ \\
\hline Teste BPG & $511.83 * * *$ & $392.32 * * *$ & $438.02 * * *$ & $126.46^{* * *}$ & $103.23 * * *$ & $113.35^{* * *}$ \\
\hline Teste Hausman & $26.58 * * *$ & $44.03 * * *$ & $40.10^{* * *}$ & $58.21 * * *$ & $86.77 * * *$ & $87.92 * * *$ \\
\hline Teste Wald & $6.6 \mathrm{e}+07 * * *$ & $3.7 \mathrm{e}+31 * * *$ & $5.9 \mathrm{e}+32 * * *$ & $6.8 \mathrm{e}+06 * * *$ & $1.8 \mathrm{e}+06^{* * *} *$ & $3.2 \mathrm{e}+06^{* * *}$ \\
\hline Teste Wooldridge & $3.318^{*}$ & $2.886^{*}$ & $2.865^{*}$ & $17.522 * * *$ & $18.948 * * *$ & $17.447 * * *$ \\
\hline
\end{tabular}

Notas: ASY1 = liquidez da ação no ano; EXP = estrutura de propriedade no fim do ano; EXP*ASY1 = interação (multiplicação) entre estrutura de propriedade no fim do ano e liquidez da ação no ano; RISC = risco (beta) da ação no ano; RENT = rentabilidade $(\mathrm{ROA})$ da empresa no ano; OCRES $=$ oportunidade de crescimento (market to book) da empresa no ano; TAM = tamanho da empresa no fim do ano; LEMP = liquidez da empresa no ano. Dividendos = modelos cuja variável de dependente é o pagamento de dividendos por ação. JSCP = modelos cuja variável dependente é o pagamento de JSCP por ação. Modelo 1 - não contempla a variável moderadora (estrutura de propriedade); Modelo 2 - contempla a variável moderadora (estrutura de propriedade); Modelo 3 - contempla a interação da variável moderadora (estrutura de propriedade) com a proxy de assimetria (liquidez da ação). $* * * * * \mathrm{e} *=$ significância estatística (p valor) a $1 \%, 5 \%$ e $10 \%$, respectivamente. Os erros padrão dos modelos cujos os testes de Wooldrigde apresentaram significância estatística inferior a 5\% foram estimados por meio da estatística Driscoll-Kraay. Todos modelos foram estimados por efeitos fixos. Fonte: Autores (2021).

Os resultados para os testes da assimetria medida pela liquidez da ação, apresentados nas Tabelas 3 e 4, não rejeitam as hipóteses desta pesquisa. A liquidez da ação apresentou relação negativa com o pagamento de dividendos e com o pagamento de JSCP. Desse modo, tendo em vista que a assimetria de informação e a liquidez da ação tem relação inversa (Albanez \& Valle, 2009; Lin et al., 2017; Spence, 1973), as empresas com maior assimetria de informação pagam mais dividendos e JSCP. Isso ocorre porque essas empresas pretendem diminuir o recurso disponível nas mãos dos gerentes e, consequentemente, os conflitos de agência (Moreiras, Tambosi Filho \& Garcia, 2012).

No entanto, a interação entre estrutura de propriedade e assimetria de informação mostra-se mais relevante para determinar o pagamento de dividendos e JSCP do que somente a assimetria. Assim, quanto maior a liquidez da ação (menor a assimetria) e maior o grau de expropriação por parte do acionista controlador, menor o pagamento de dividendos e JSCP por ação. Em outras palavras, em empresas com menor grau de assimetria de informações em que o principal acionista tem mais controle sobre a empresa e menos direito sobre o fluxo de caixa, há menores pagamentos aos investidores. Nesse contexto, exercendo forte influência sobre as decisões dos gestores (La Porta et al., 2000), os acionistas majoritários expropriam os minoritários diminuindo a remuneração por meio de pagamentos de dividendos e JSCP. 
Em relação às demais variáveis, os resultados mostraram que o risco da empresa interfere significativamente apenas no pagamento de dividendos. Assim, empresas com maiores riscos são menos propensas a pagar dividendos (Bernardo \& Ikeda, 2013; C. A. B. Forti et al., 2015; Lin et al., 2017; Ramli, 2010; Rozeff, 1982; Rosa, Araújo \& Rogers, 2021). A relação entre risco e JSCP, no entanto, não apresentou significância estatística. Como o pagamento de JSCP, em comparação com o pagamento de dividendos, representa vantagens fiscais para a empresa (Boulton et al., 2012) a propensão de empresas com maior risco de não remunerar o acionista pode ser atenuada com a possibilidade de pagamento via JSCP. Dessa forma, a empresa com maior risco relutaria em realizar pagamentos ao investidor, mas teria um estímulo (benefício fiscal) para remunerar o acionista e evitar um declínio nos preços das ações (Rajverma et al., 2019). Os resultados, apresentados na Tabela 4, corroboram com essa afirmação.

Conforme Tabela 4, em empresas com maior risco, a diferença entre o valor pago pelo preço ação via dividendos e o pago em relação ao preço ação via JSCP é menor. Ou seja, quanto maior o risco da empresa, menos propensão a pagar dividendos em comparação com o pagamento via JSCP. No entanto, com a inclusão da variável moderadora (estrutura de propriedade), esta relação deixa de ser estatisticamente significativa. Dessa forma, assim como ocorre com a assimetria de informação, a interação entre assimetria e estrutural de propriedade passa a ser mais relevante do que o risco da empresa para a diferença entre o valor de dividendos pago por ação e o valor de JSCP pago por ação.

Tabela 4. Resultados para pagamentos totais e diferença entre dividendos e JSCP - liquidez da ação como proxy de assimetria

\begin{tabular}{|c|c|c|c|c|c|c|}
\hline \multirow[b]{2}{*}{ Variável } & \multicolumn{3}{|c|}{ Pagamentos totais } & \multicolumn{3}{|c|}{ Dividendos - JSCP } \\
\hline & Modelo 1 & Modelo 2 & Modelo 3 & Modelo 1 & Modelo 2 & Modelo 3 \\
\hline ASY1 & $-0.925 * * *$ & $-0.942 * *$ & -0.096 & 0.049 & 0.375 & 0.939 \\
\hline EXP & & -0.029 & & & -0.016 & \\
\hline EXP*ASY1 & & & $-0.078 * * *$ & & & $-0.053 *$ \\
\hline RISC & $-0.534 * *$ & $-0.376^{*}$ & -0.352 & $-0.657 * *$ & -0.412 & -0.396 \\
\hline RENT & -0.013 & $0.063 * *$ & $0.062 * *$ & -0.032 & 0.041 & 0.041 \\
\hline OCRES & $-0.581 * * *$ & $-0.563 * * *$ & $-0.566 * * *$ & $-0.460 * * *$ & $-0.372 * * *$ & $-0.374 * * *$ \\
\hline TAM & $-2.390 * *$ & $-2.360 * * *$ & $-2.412 * * *$ & $-3.649 * * *$ & $-3.258 * * *$ & $-3.307 * * *$ \\
\hline LEMP & $-0.098 *$ & -0.054 & -0.056 & $-0.143 * * *$ & $-0.091 * * *$ & $-0.092 * * *$ \\
\hline CONS & $23.815^{* * *}$ & $23.258 * * *$ & $23.117 * * *$ & $29.918 * * *$ & $26.265 * * *$ & $26.329 * * *$ \\
\hline VIF (médio) & 1.28 & 1.27 & 1.51 & 1.28 & 1.27 & 1.51 \\
\hline Teste F & $8.69 * * *$ & $8.04 * * *$ & $9.37 * * *$ & $4.42 * * *$ & $3.89 * * *$ & $3.49 * *$ \\
\hline $\mathrm{R}^{2}$ & 0.047 & 0.043 & 0.045 & 0.037 & 0.022 & 0.023 \\
\hline $\mathrm{n}$ & 2961 & 2485 & 2485 & 2960 & 2484 & 2484 \\
\hline Teste Chow & $3.55 * * *$ & $3.73 * * *$ & $3.91 * * *$ & $3.62 * * *$ & $3.72 * * *$ & $3.85 * * *$ \\
\hline Teste BPG & $704.15 * * *$ & $547.96 * * *$ & $622.45 * * *$ & $752.07 * * *$ & $571.34 * * *$ & $628.40 * * *$ \\
\hline Teste Hausman & $29.70 * * *$ & $52.52 * * *$ & $49.78 * * *$ & $29.58 * * *$ & $40.81 * * *$ & $39.33 * * *$ \\
\hline Teste Wald & $2.5 \mathrm{e}+32 * * *$ & $9.0 \mathrm{e}+31 * * *$ & $5.9 \mathrm{e}+31 * * *$ & $9.6 \mathrm{e}+06^{* * *}$ & $3.6 \mathrm{e}+06 * * *$ & $1.9 \mathrm{e}+31 * * *$ \\
\hline Teste Wooldridge & $4.734 * *$ & $3.065^{*}$ & $3.062 *$ & $15.802 * * *$ & $10.429 * * *$ & $10.567 * * *$ \\
\hline
\end{tabular}

Notas: ASY1 = liquidez da ação no ano; EXP = estrutura de propriedade no fim do ano; EXP*ASY1 = interação (multiplicação) entre estrutura de propriedade no fim do ano e liquidez da ação no ano; RISC = risco (beta) da ação no ano; RENT = rentabilidade (ROA) da empresa no ano; OCRES = oportunidade de crescimento (market to book) da empresa no ano; TAM = tamanho da empresa no fim do ano; LEMP = liquidez da empresa no ano. Pagamentos totais = modelos cuja variável de dependente é o pagamento total (dividendos + JSCP) por ação. DIF = modelos cuja variável dependente é a diferença entre o pagamento de dividendos por ação e o pagamento de JSCP por ação. Modelo 1 - não contempla a variável moderadora (estrutura de propriedade); Modelo 2 - contempla a variável moderadora (estrutura de propriedade); Modelo 3 - contempla a interação da variável moderadora (estrutura de propriedade) com a proxy de assimetria (liquidez da ação). $* * *, * * \mathrm{e}^{*}=$ significância estatística (p valor) a $1 \%, 5 \%$ e $10 \%$, respectivamente. Os erros padrão dos modelos cujos os testes de Wooldrigde apresentaram significância estatística inferior a 5\% foram estimados por meio da estatística Driscoll-Kraay. Todos modelos foram estimados por efeitos fixos. Fonte: Autores (2021) 
Não obstante, a estrutura de propriedade modera, também, a relação entre rentabilidade e pagamento de dividendos. Empresas com maior rentabilidade sobre o ativo pagam mais dividendos (Forti et al, 2015; Lin et al, 2017). Essa relação também é verificada para JSCP e pagamento total. Entretanto, antes da inclusão da variável estrutura de propriedade (Modelo 1 das tabelas), a rentabilidade não apresentou relação estatisticamente significativa com o pagamento de dividendos e JSCP e com o pagamento total. Ademais, a rentabilidade não influencia de forma significativa a diferença entre pagamento de dividendos e pagamento de JSCP.

Em todos os modelos, a oportunidade de crescimento apresentou relação negativa com o pagamento de dividendos e JSCP. Como o pagamento de dividendos pode sinalizar ao mercado que a empresa não tem boas opções de investimento (Allen \& Michaely, 2003), faz sentido que empresas com maiores oportunidades de crescimento paguem menos dividendos e utilizem seus recursos para financiar projetos de investimento (Lin et al., 2017).

Em relação à liquidez geral da empresa, de forma geral, os resultados indicam que esse é um fator que afeta mais o pagamento de dividendos do que JSCP. Empresas com maior grau de liquidez pagam menos dividendos (Ferreira Jr et al., 2010; Lin et al., 2017). Desse modo, infere-se que empresas mais líquidas podem estabelecer uma melhor política de dividendos que maximize o valor da empresa sem gerar efeito negativo nas metas futuras de investimento e no fluxo de caixa da empresa (Topak \& Dereli, 2018), não sendo, portanto, pressionadas a pagar dividendos para evitar declínio no preço das ações. Outrossim, a liquidez da empresa tem relação negativa com a diferença entre o pagamento de dividendos sobre ação e o pagamento de JSCP sobre o preço ação. Esses resultados estão evidenciados, também, nas Tabelas 5 e 6. 
Tabela 5. Resultados para pagamento de dividendos e JSCP - volatilidade da ação como proxy de assimetria.

\begin{tabular}{|c|c|c|c|c|c|c|}
\hline \multirow[b]{2}{*}{ Variável } & \multicolumn{3}{|c|}{ Pagamentos totais } & \multicolumn{3}{|c|}{ Dividendos - JSCP } \\
\hline & Modelo 1 & Modelo 2 & Modelo 3 & Modelo 1 & Modelo 2 & Modelo 3 \\
\hline ASY2 & $0.044 * * *$ & $0.030 * *$ & $0.029 * *$ & $0.025 * *$ & $0.030 * *$ & $0.033 * *$ \\
\hline EXP & & -0.018 & & & -0.024 & \\
\hline EXP*ASY2 & & & 0.000 & & & 0.000 \\
\hline RISC & $-1.123 * * *$ & $-0.852 * * *$ & $-0.844 * * *$ & $-0.356^{*}$ & $-0.450 *$ & $-0.452 *$ \\
\hline RENT & -0.023 & $0.064 *$ & $0.064 *$ & -0.012 & -0.013 & -0.012 \\
\hline OCRES & $-0.538 * * *$ & $-0.509 * * *$ & $-0.508 * * *$ & $-0.308 * * *$ & $-0.326^{* * *}$ & $-0.321 * * *$ \\
\hline TAM & $-2.602 * * *$ & $-2.610 * *$ & $-2.517 * *$ & $-2.488 * *$ & $-2.701 * *$ & $-2.601 * *$ \\
\hline LEMP & $-0.110 * * *$ & $-0.074 * *$ & $-0.076^{* *}$ & 0.000 & 0.000 & 0.000 \\
\hline CONS & $23.243 * * *$ & $23.076 * * *$ & $22.177 * * *$ & $19.966 * * *$ & $21.678 * * *$ & $20.627 * * *$ \\
\hline VIF (médio) & 1.14 & 1.15 & 1.24 & 1.19 & 1.18 & 1.28 \\
\hline Teste F & $9.38 * * *$ & $6.11 * * *$ & $6.55^{* * *}$ & $6.63 * * *$ & $4.40 * * *$ & $4.66^{* * *}$ \\
\hline $\mathrm{R}^{2}$ & 0.060 & 0.041 & 0.041 & 0.142 & 0.162 & 0.16 \\
\hline $\mathrm{n}$ & 2634 & 2190 & 2190 & 1163 & 1071 & 1071 \\
\hline Teste Chow & $3.13 * * *$ & $3.32 * * *$ & $3.33 * * *$ & $3.05^{* * *}$ & $2.83 * * *$ & $2.83 * * *$ \\
\hline Teste BPG & $506.15^{* * *}$ & $399.10 * * *$ & $404.48^{* * *}$ & $136.01 * * *$ & $125.23 * * *$ & $125.23 * * *$ \\
\hline Teste Hausman & $24.74 * * *$ & $39.83 * * *$ & $38.51 * * *$ & $35.74 * * *$ & $50.18 * * *$ & $50.07 * * *$ \\
\hline Teste Wald & $1.9 \mathrm{e}+33^{* * *} *$ & $4.1 \mathrm{e}+32 * * *$ & $6.7 \mathrm{e}+32 * * *$ & $3.0 \mathrm{e}+28 * * *$ & $2.3 \mathrm{e}+29 * * *$ & $1.8 \mathrm{e}+29 * * *$ \\
\hline Teste Wooldridge & 2.690 & $3.001 *$ & $3.139 *$ & $16.623 * * *$ & $15.453 * * *$ & $15.312 * * *$ \\
\hline
\end{tabular}

Notas: ASY2 = volatilidade da ação no ano; EXP = estrutura de propriedade no fim do ano; EXP*ASY2 = interação (multiplicação) entre estrutura de propriedade no fim do ano e volatilidade da ação no ano; RISC = risco (beta) da ação no ano; RENT = rentabilidade (ROA) da empresa no ano; OCRES = oportunidade de crescimento (market to book) da empresa no ano; TAM = tamanho da empresa no fim do ano; LEMP = liquidez da empresa no ano. Dividendos = modelos cuja variável de dependente é o pagamento de dividendos por ação . JSCP = modelos cuja variável dependente é o pagamento de JSCP por ação. Modelo 1 - não contempla a variável moderadora (estrutura de propriedade); Modelo 2 - contempla a variável moderadora (estrutura de propriedade); Modelo 3 - contempla a interação da variável moderadora (estrutura de propriedade) com a proxy de assimetria (liquidez da ação). ***, ** e * = significância estatística (p valor) a $1 \%$, $5 \%$ e $10 \%$, respectivamente. Os erros padrão dos modelos cujos os testes de Wooldrigde apresentaram significância estatística inferior a $5 \%$ foram estimados por meio da estatística Driscoll-Kraay. Todos modelos foram estimados por efeitos fixos. Fonte: Autores (2021)

Na Tabela 5, assim como na Tabela 6, verifica-se que a assimetria de informação, medida pela volatilidade das ações da empresa, apresenta relação positiva com o pagamento de dividendos. Dessa forma, por meio de outra proxy, a hipótese de relação positiva entre assimetria de informação e remuneração ao acionista não foi rejeitada. No entanto, de forma geral, a estrutura de propriedade não moderou a relação entre volatilidade (proxy de assimetria de informação) e a remuneração aos acionistas.

Assim sendo, analisando neste contexto, as empresas com maior assimetria pagam mais dividendos para mitigar o conflito de agência (Lin et al., 2017; Moreiras et al., 2012) e a estrutura de propriedade é estatisticamente insignificante nessa relação. Nessa perspectiva, empresas com maior assimetria de informação remuneram mais o acionista independentemente do grau de expropriação por parte do acionista majoritário.

Contudo, conforme Tabela 6, a interação entre assimetria (medida por volatilidade) e estrutura de propriedade diminui a relevância da assimetria na diferença entre o valor pago de dividendos por ação e o valor de JSCP pago por ação. Ou seja, em empresas com maior assimetria, a diferença entre o valor pago via dividendos e o pago via JSCP é maior, paga-se mais dividendos em comparação com JSCP. No entanto, quando se considera o fator de interação entre estrutura de propriedade e assimetria, esta deixa de ter relação estatisticamente significativa com a diferença do valor pago por meio de dividendos e JSCP. 
Tabela 6. Resultados para pagamentos totais e diferença entre dividendos e JSCP - volatilidade da ação como proxy de assimetria

\begin{tabular}{|c|c|c|c|c|c|c|}
\hline \multirow[b]{2}{*}{ Variável } & \multicolumn{3}{|c|}{ Pagamentos totais } & \multicolumn{3}{|c|}{ Dividendos - JSCP } \\
\hline & Modelo 1 & Modelo 2 & Modelo 3 & Modelo 1 & Modelo 2 & Modelo 3 \\
\hline ASY2 & $0.043 * * *$ & $0.031 * *$ & $0.032 * * *$ & $0.038 * * *$ & $0.022 * *$ & 0.021 \\
\hline EXP & & -0.026 & & & -0.019 & \\
\hline EXP*ASY2 & & & 0.000 & & & 0.000 \\
\hline RISC & $-1.000 * * *$ & $-0.732 * * *$ & $-0.732 * * *$ & $-1.023 * * *$ & $-0.616^{* *}$ & $-0.608 * *$ \\
\hline RENT & -0.002 & $0.065^{* *}$ & $0.065 * *$ & -0.022 & 0.047 & 0.047 \\
\hline OCRES & $-0.561 * * *$ & $-0.550 * * *$ & $-0.548 * * *$ & $-0.425 * * *$ & $-0.345 * * *$ & $-0.344 * * *$ \\
\hline TAM & $-2.080 * *$ & $-2.225 * *$ & $-2.108 * *$ & $-2.999 * *$ & $-2.656 * *$ & $-2.561 * *$ \\
\hline LEMP & $-0.081 *$ & -0.048 & -0.045 & $-0.126 * * *$ & $-0.085 * * *$ & $-0.086 * * *$ \\
\hline CONS & $20.025 * * *$ & $21.066^{* * *}$ & $19.852 * * *$ & $24.258 * * *$ & $21.644 * * *$ & 20.716 \\
\hline VIF (médio) & 1.14 & 1.15 & 1.24 & 1.14 & 1.15 & 1.24 \\
\hline Teste F & $7.97 * * *$ & $8.37 * * *$ & $8.95 * * *$ & $5.24 * * *$ & $4.49 * * *$ & $3.86 * * *$ \\
\hline $\mathrm{R}^{2}$ & 0.059 & 0.048 & 0.047 & 0.046 & 0.025 & 0.024 \\
\hline$n$ & 2961 & 2485 & 2485 & 2960 & 2484 & 2484 \\
\hline Teste Chow & $3.52 * * *$ & $3.69 * * *$ & $3.71 * * *$ & $3.58 * * *$ & $3.68 * * *$ & $3.70 * * *$ \\
\hline Teste BPG & $690.89 * * *$ & $557.02 * * *$ & $557.02 * * *$ & $772.80 * * *$ & $610.56^{* * *}$ & $622.72 * * *$ \\
\hline Teste Hausman & $27.69 * * *$ & $44.59 * * *$ & $42.14 * * *$ & $29.90 * * *$ & $41.80 * * *$ & $40.52 * * *$ \\
\hline Teste Wald & $2.1 \mathrm{e}+33 * * *$ & $1.5 \mathrm{e}+32 * * *$ & $1.5 \mathrm{e}+32 * * *$ & $2.1 \mathrm{e}+32 * * *$ & $2.0 \mathrm{e}+33 * * *$ & $6.6 \mathrm{e}+29 * * *$ \\
\hline Teste Wooldridge & $4.030 * *$ & $3.252 *$ & $3.291^{*}$ & $14.932 * * *$ & $10.569 * * *$ & $10.716^{* * *}$ \\
\hline
\end{tabular}

Notas: ASY2 = volatilidade da ação no ano; EXP = estrutura de propriedade no fim do ano; EXP*ASY2 = interação (multiplicação) entre estrutura de propriedade no fim do ano e volatilidade da ação no ano; RISC = risco (beta) da ação no ano; RENT = rentabilidade (ROA) da empresa no ano; OCRES = oportunidade de crescimento (market to book) da empresa no ano; TAM = tamanho da empresa no fim do ano; LEMP = liquidez da empresa no ano. Pagamentos totais $=$ modelos cuja variável de dependente é o pagamento total (dividendos + JSCP) por ação. DIV-JSCP = modelos cuja variável dependente é a diferença entre o pagamento de dividendos por ação e o pagamento de JSCP por ação. Modelo 1 - não contempla a variável moderadora (estrutura de propriedade); Modelo 2 - contempla a variável moderadora (estrutura de propriedade); Modelo 3 - contempla a interação da variável moderadora (estrutura de propriedade) com a proxy de assimetria (liquidez da ação). ***, ** e $*$ significância estatística (p valor) a $1 \%, 5 \%$ e $10 \%$, respectivamente. Os erros padrão dos modelos cujos os testes de Wooldrigde apresentaram significância estatística inferior a 5\% foram estimados por meio da estatística Driscoll-Kraay. Todos modelos foram estimados por efeitos fixos. Fonte: Autores (2021)

A Tabela 6 mostra, ainda, que o tamanho da empresa tem relação negativa com remuneração ao acionista. Esse resultado foi verificado em relação a todas as formas de remuneração, seja por meio de dividendos, JSCP ou a soma dos dois. Desse modo, quanto maior o tamanho da empresa, menor a remuneração ao acionista, em contraposição à literatura (Rogers, Araújo \& Rogers, 2021; Forti et al., 2015; Heineberg \& Procianoy, 2003; Iquiapaza et al., 2006; Lee \& Xiao, 2011; Mota \& Junior, 2007; Ramli, 2010; Wei et al., 2004). Os resultados mostraram, também, que quanto maior o tamanho da empresa, menor a diferença entre o valor pago de dividendos e o valor pago por meio de JSCP.

\section{Considerações Finais}

Esta pesquisa analisou o efeito moderador da estrutura de propriedade na relação entre assimetria de informação e remuneração ao acionista. A assimetria de informação foi representada pelas proxies liquidez da ação e volatilidade, em modelos de regressão distintos. A estrutura de propriedade foi definida pelo grau de expropriação por parte do acionista majoritário, medida pela diferença entre o direito de controle e o direito sobre o fluxo de caixa, por parte do acionista controlador. Não obstante, testou-se a hipótese de que a assimetria de informação afeta positivamente a remuneração ao acionista. Essa hipótese $\left(H_{l}\right)$ não foi rejeitada. 
Em relação ao efeito moderador da estrutura de propriedade, os resultados não foram consensuais $\left(H_{2}\right)$. Quando medida por liquidez da ação, a assimetria de informação apresentou relação positiva e estatisticamente significativa com a remuneração ao acionista apenas nos modelos que não consideravam a interação do efeito moderador. Nesse sentido, a interação moderou o efeito da assimetria na remuneração ao acionista, de modo que o esse fator combinado tem maior influência na decisão de remuneração ao acionista, enquanto a assimetria por si só passa a ser estatisticamente irrelevante.

Entretanto, considerando a volatilidade da ação como medida de assimetria, a interação entre os fatores não moderou a relação entre assimetria e a remuneração ao acionista. Desse modo, a assimetria manteve relação positiva e estatisticamente significativa em relação à remuneração, seja via dividendos, JSCP ou a soma dos dois. Não obstante, além de ser estatisticamente irrelevante, a interação entre a estrutura de propriedade e a assimetria (medida pela volatilidade) apresentou pouco efeito prático, haja vista o coeficiente da variável combinada ter sido muito baixo.

Assim sendo, o trabalho contribui com a literatura e com os interessados no mercado de ações brasileiro ao apresentar, de diferentes perspectivas, o efeito moderador da estrutura de propriedade na relação entre assimetria de informação e remuneração ao acionista. Além disso, esta pesquisa analisou de forma separada a remuneração ao acionista. Tem sido comum nos estudos sobre o mercado brasileiro considerar conjuntamente dividendos e JSCP. No entanto, este estudo evidenciou que dividendos e JSCP são afetados de formas diferentes por alguns fatores, tais como rentabilidade, risco e liquidez da empresa.

Esta pesquisa apresenta a limitação inerente aos estudos sobre assimetria de informação: a dificuldade para definir uma proxy para esse fator. Outros estudos consideraram mais de um fator para medir assimetria devido à inexistência de consenso no melhor medidor dessa variável. Dessa forma, sugere-se que, em pesquisas futuras, sejam testadas outras variáveis proxies de assimetria que não foram consideradas nesta pesquisa. $\mathrm{O}$ desvio padrão da previsão dos analistas pode ser uma medida interessante para a variável, utilizada em alguns estudos sobre outros mercados, mas, neste momento, apresenta a limitação de reduzir consideravelmente a amostra em razão do baixo número de empresas do mercado brasileiro cobertas por analistas.

\section{Referências}

Al-Najjar, B. (2011). The inter-relationship between capital structure and dividend policy: empirical evidence from Jordanian data. International Review of Applied Economics, 25(2), 209-224. https://doi.org/10.1080/02692171.2010.483464

Albanez, T., \& Valle, M. R. do. (2009). Impactos da assimetria de informação na estrutura de capital de empresas brasileiras abertas 1 Impacts of information asymmetry on the capital structure. Revista de Contabilidade e Finanças, v. 20(33), 6-27.

Allen, F., \& Michaely, R. (2003). Handbook of the Economics of Finance. In G. M. Constantinides, M. Harris, \& R. Stulz (Eds.), Elsevier (pp. 337-429). Elsevier B.V. Retrieved from http://portal.lscmalta.edu.mt/resources/mod/folder/view.php?id=3700

Ben-Nasr, H. (2015). Government Ownership and Dividend Policy: Evidence from Newly Privatised Firms. Journal of Business Finance and Accounting, 42(5-6), 665-704. https://doi.org/10.1111/jbfa.12115

Bernardo, H. P., \& Ikeda, R. H. (2013). O Enigma Dos Dividendos E O Risco Sistemático. Revista Universo Contábil, 9(1), 104-120. https://doi.org/10.4270/ruc.2013106

Boulton, T. J., Braga-Alves, M. V., \& Shastri, K. (2012). Payout policy in Brazil: Dividends versus interest on equity. Journal of Corporate Finance, 18(4), 968-979. https://doi.org/10.1016/j.jcorpfin.2011.09.004

Duqi, A., Jaafar, A., \& Warsame, M. H. (2019). Payout policy and ownership structure: The case of Islamic and conventional banks. The British Accounting Review. https://doi.org/10.1016/j.bar.2019.03.001

Ferreira Jr, W. O., Nakamura, W. T., Martin, D. M. L., \& Bastos, D. D. (2010). Evidências Empíricas Dos Fatores Determinantes Das Políticas De Dividendos Das Firmas Listadas Na Bovespa. FACEF Pesquisa - Desenvolvimento e Gestão, 13(2), 190-203.

Forti, C. A. B., Peixoto, F. M., \& Alves, D. L. e. (2015). Determinant Factors of Dividend Payments in Brazil. Revista Contabilidade \& Finanças, 26(68), 167-180. https://doi.org/10.1590/1808-057x201512260

Forti, C., \& Schiozer, R. F. (2015). Bank dividends and signaling to information-sensitive depositors. Journal of Banking and Finance, 56(February), 1-11. https://doi.org/10.1016/j.jbankfin.2015.02.011

Futema, M. S., Basso, L. F. C., \& Kayo, E. K. (2009). Estrutura de capital, dividendos e juros sobre o capital próprio: testes no Brasil. Revista Contabilidade 
\& Finanças, 20(49), 44-62. https://doi.org/10.1590/S1519-70772009000100004

Gugler, K. (2003). Corporate governance, dividend payout policy, and the interrelation between dividends, R\&amp;D, and capital investment. Journal of Banking \& Finance, 27(7), 1297-1321. https://doi.org/10.1016/S0378-4266(02)00258-3

Heineberg, R., \& Procianoy, J. L. (2003). Aspectos determinantes do pagamento de proventos em dinheiro das empresas com ações negociadas na Bovespa. In Encontro Nacional da Associação Nacional dos Programas de Pós-graduação em Administração, 27, 2003, Atibaia. Atibaia: ANPAD. https://doi.org/10.1017/CBO9781107415324.004

Holanda, A. P., \& Coelho, A. C. D. (2012). Dividendos e efeito clientela: evidências no mercado brasileiro. Revista de Administração de Empresas, 52(4), 448-463. https://doi.org/10.1590/S0034-75902012000400007

Huddart, S. (1993). The Effect of a Large Shareholder on Corporate Value. Management Science, 39(11), 1407-1421. https://doi.org/10.1287/mnsc.39.11.1407

Iquiapaza, R., Lamounier, W., \& Amaral, H. (2006). Asymmetric Information and Dividends Payment at Bovespa. Munich Personal RePEc Archive, (1673). Retrieved from https://mpra.ub.uni-muenchen.de/1673/

Jensen, M. C., \& Meckling, W. H. (1976). Theory of the firm: Managerial behavior, agency costs and ownership structure. Journal of Financial Economics.

Kumar, A., \& Vergara-Alert, C. (2018). The Effect of Financial Flexibility on Payout Policy. Journal of Financial and Quantitative Analysis, 1-27. https://doi.org/10.1017/S002210901800114X

La Porta, R., Lopez-De-Silanes, F., Shleifer, A., \& Vishny, R. W. (2000). Agency Problems and Dividend Policies Around the World. The Journal of Finance, 55(1), 1-33. https://doi.org/10.2307/222549

Lee, C.-W. J., \& Xiao, X. (2011). Tunneling Dividends. SSRN Electronic Journal, (03). https://doi.org/10.2139/ssrn.693361

Leuz, C., Nanda, D., \& Wysocki, P. D. (2003). Earnings management and investor protection: an international comparison. Journal of Financial Economics, 69, 505-527. https://doi.org/10.1016/S0304-405X(03)00121-1

Lin, T., Chen, Y., \& Tsai, H. (2017). The relationship among information asymmetry, dividend policy and ownership structure. Finance Research Letters, 20, 1-12. https://doi.org/10.1016/j.frl.2016.06.008

Marques, B. S., Perpétuo, A. A. G., \& Rocha, D. M. (2020). Análise bibliométrica sobre os estudos de dividendos no Brasil. Research, Society and Development, 9(8). http://dx.doi.org/10.33448/rsd-v9i8.5658

Martins, A. I., \& Famá, R. (2012). O que revelam os estudos realizados no Brasil sobre política de dividendos? Revista de Administração de Empresas, 52(1), 24-39. https://doi.org/10.1590/S0034-75902012000100003

Martins, T. C., \& Novaes, W. (2012). Mandatory dividend rules: Do they make it harder for firms to invest? Journal of Corporate Finance, 18(4), 953-967. https://doi.org/10.1016/j.jcorpfin.2012.05.002

Maug, E. (1998). Large shareholders as monitors: Is there a trade-off between liquidity and control? Journal of Finance, 53(1), 65-98. https://doi.org/10.1111/0022-1082.35053

Moreiras, L. M. F., Tambosi Filho, E., \& Garcia, F. G. (2012). Dividendos e informação assimétrica: análise do novo mercado. Revista de Administração, 47'(4), 671-682. https://doi.org/10.5700/rausp1066

Mota, D. C., \& Junior, W. E. (2007). Dividendos, juros sobre capital próprio e recompra de ações: um estudo empírico sobre a política de distribuição no Brasil. In XXXI Encontro da ANPAD (pp. 1-16). Rio de Janeiro: EnANPAD.

Ofer, A. R., \& Siegel, D. R. (1987). Corporate Financial Policy, Information, and Market Expectations: An Empirical Investigation of Dividends. The Journal of Finance, 42(4), 889-911.

La Porta, R., Lopez-de-Silanes, F., \& Shleifer, A. (1999). Corporate Ownership Around the World. The Journal of Finance, 54(2), $471-517$.

Pereira, A. S., Shitsuka, D. M., Parreira, F. J., \& Shitsuka, R. (2018). Metodologia da pesquisa científica. Editora UAB/NTE/UFSM. Santa Maria-RS

Procianoy, J. L., \& Verdi, R. S. (2009). Dividend Clientele, new insights, and new questions: the brazilian case. Revista de Administração de Empresas Eletrônica, 8(1), Art. 1. https://doi.org/10.1017/CBO9781107415324.004

Rajverma, A. K., Misra, A. K., Mohapatra, S., \& Chandra, A. (2019). Impact of ownership structure and dividend on firm performance and firm risk. Managerial Finance, 45(8), 1041-1061. https://doi.org/10.1108/MF-09-2018-0443

Ramli, N. M. (2010). Ownership structure and dividend policy: Evidence from Italian firms. International Review of Business Research Papers, 6(1), 170180. https://doi.org/10.1080/13518470500249365

Rosa, A. A., Araújo, F. B. B. \& Rogers, P. (2021). Política de dividendos e volatilidade do preço das empresas brasileiras de capital aberto. Research, Society and Development, 10(4). http://dx.doi.org/10.33448/rsd-v10i4.14210

Rozeff, M. S. (1982). Growth, beta and agency costs as determinants of dividend payout ratios. The Journal of Financial Research, 5(3), 249-259.

Santos, A. Dos. (2007). Quem está pagando juros sobre capital próprio no Brasil? Revista Contabilidade \& Finanças, 18(6), 33-44. https://doi.org/10.1590/S1519-70772007000300004

Silva, A. L. C. da. (2004). Governanca corporativa, valor, alavancagem e política de dividendos nas empresas brasileiras. Revista de Administração, 39(4), $348-361$. 
Research, Society and Development, v. 10, n. 5, e52010515293, 2021

(CC BY 4.0) | ISSN 2525-3409 | DOI: http://dx.doi.org/10.33448/rsd-v10i5.15293

Spence, M. (1973). Job Market Signaling. The Quarterly Journal of Economics, 87(3), 355-374.

Tomaz, N. C. B. (2017). Relação entre a distribuição de dividendos e o preço da ação das empresas listadas na B3. Monografia. Universidade Federal da Paraíba, João Pessoa.

Topak, M. S., \& Dereli, H. (2018). The Effect of Dividend Policy on Stock Price: Evidence from An Emerging Country. Journal of Business Research - Turk, 10(4), 1186-1197. https://doi.org/10.20491/isarder.2018.569

Vancin, D. F., \& Procianoy, J. L. (2016). Os Fatores Determinantes do Pagamento de Dividendos : o Efeito do Obrigatório Mínimo Legal e Contratual nas Empresas Brasileiras. Brazilian Review of Finance, 14(1), 89-123.

Vogelsang, T. J. (2012). Heteroskedasticity, autocorrelation, and spatial correlation robust inference in linear panel models with fixed-effects. Journal of Econometrics, 166(2), 303-319. https://doi.org/10.1016/j.jeconom.2011.10.001

Wei, J. G., Zhang, W., \& Xiao, J. Z. (2004). Dividend Payment and Ownership Structure in China. Advances in Financial Economics, 9, 187-219. https://doi.org/10.1016/S1569-3732(04)09008-5 